\title{
Hybrid structural methods to probe atomic features of the Type III Secretion Injectisome of Pathogenic Bacteria
}

Natalie Strynadka

University of British Columbia, United States

Bacteria have evolved several sophisticated assemblies to transport proteins across their biological membranes, including those required specifically for pathogenicity. Recent advances in our understanding of the molecular details governing the molecular action of these protein secretion systems has benefited from an integrated toolbox of x-ray crystallography, NMR, mass spectroscopy, molecular modelling and increasingly and most dramatically, cryo electron microscopy. A syringe like nanoassembly, the Type III Secretion system, injects multiple virulence "effector" proteins from the bacterial cytosol through to the infected host cell. These effectors manipulate host cell processes in varying ways to the benefit and subsequent pathogenicity of the bacteria. An essential element of disease in several of the most notorious Gram negative bacterial pathogens including the causative agents of food and water borne disease, hospital sepsis, cholera, typhoid fever, bubonic plague and sexually transmitted disease, a molecular understanding of the Type III Secretion systems being garnered from these structural studies provides the foundation for the development of new classes of antibacterials and vaccines to combat infection in the clinic and community. Highlights of recent advances in our structure/function analysis of the multi-membrane spanning Type III Secretion system "injectisome" will be presented emphasizing cryoEM focused refinements of the symmetry mismatched components of the core Type III Secretion System basal body complex spanning the inner through outer membranes of the prototypical Gram negative Salmonella typhimurium bacterial variant. These studies highlight a remarkable set of unexpected interactions including localized recruitment of protomers to allow symmetric coupling interactions between the inner and outer membrane components and a nanodisc like interaction of the inner membrane rings with the multicomponent export apparatus complex "gate", T3SS proteins previously predicted to be membrane spanning in nature, but clearly sitting atop the membrane bilayer in the assembled structures.

\section{References}

1: Lyons BJE, Atkinson CE, Deng W, Serapio-Palacios A, Finlay BB, Strynadka NCJ. Cryo-EM structure of the EspA filament from enteropathogenic Escherichia coli:

revealing the mechanism of effector translocation in the T3SS. Structure. 2021 Jan 13:S0969-2126(20)30476-7.

2: Majewski DD, Lyons BJE, Atkinson CE, Strynadka NCJ. Cryo-EM analysis of the SctV cytosolic domain from the enteropathogenic E. coli T3SS injectisome. J Struct Biol. 2020 Dec 1;212(3):107660.

3: Hu J, Worrall LJ, Strynadka NC. Towards capture of dynamic assembly and action of the T3SS at near atomic resolution. Curr Opin Struct Biol. 2020

Apr;61:71-78.

4: Lyons BJE, Strynadka NCJ. On the road to structure-based development of antivirulence therapeutics targeting the type III secretion system injectisome.

Medchemcomm. 2019 Jun 20;10(8):1273-1289.

5: Hu J, Worrall LJ, Vuckovic M, Hong C, Deng W, Atkinson CE, Brett Finlay B, Yu

$\mathrm{Z}$, Strynadka NCJ. T3S injectisome needle complex structures in four distinct states reveal the basis of membrane coupling and assembly. Nat Microbiol. 2019 Nov;4(11):2010-2019. 
6: Majewski DD, Worrall LJ, Hong C, Atkinson CE, Vuckovic M, Watanabe N, Yu Z, Strynadka NCJ. Cryo-EM structure of the homohexameric T3SS ATPase-central stalk complex reveals rotary ATPase-like asymmetry. Nat Commun. 2019 Feb 7;10(1):626. 7: Hu J, Worrall LJ, Hong C, Vuckovic M, Atkinson CE, Caveney N, Yu Z, Strynadka NCJ. Cryo-EM analysis of the T3S injectisome reveals the structure of the needle and open secretin. Nat Commun. 2018 Sep 21;9(1):3840.

8: Worrall LJ, Hong C, Vuckovic M, Deng W, Bergeron JRC, Majewski DD, Huang RK, Spreter T, Finlay BB, Yu Z, Strynadka NCJ. Near-atomic-resolution cryo-EM analysis of the Salmonella T3S injectisome basal body. Nature. 2016 Dec 22;540(7634):597-601. 